Check for updates

Cite this: J. Mater. Chem. C, 2020 8, 8846

Received 2nd May 2020,

Accepted 2nd June 2020

DOI: $10.1039 / \mathrm{dOtc0} 2149 \mathrm{k}$

rsc.li/materials-c

\title{
Small molecule additive for low-power accumulation mode organic electrochemical transistors $\dagger$
}

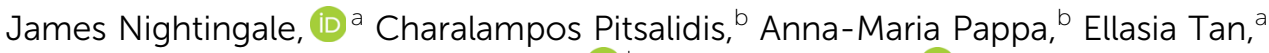 \\ Katherine Stewart, ${ }^{a}$ Róisín M. Owens (D) $^{\mathrm{b}}$ and Ji-Seon Kim (D) *a
}

\begin{abstract}
Low-voltage operation in accumulation mode organic electrochemical transistors (OECTs) is essential for biosensing applications and for potential use with low-voltage portable power supplies. Here, we employ a small molecule additive, dodecylbenzenesulfonate (DBSA), by adding it to the electrolyte in OECTs to improve the device performance. We find that DBSA lowers the operation voltage, increases the ON current, and increases the transconductance of the device. Such improvements are found for a range of p-type polymers including P3HT, PBTTT and DPPT-TT which have different electronic and structural properties. To investigate the device operational mechanism modulated by DBSA, we directly probe the molecular structure changes of three polymers upon charge injection (i.e. polaron formation) and correlate them to polaron density and OECT performance. We find that the electrolyte mixture (containing DBSA) enhances the electrochemical doping of the polymer by lowering the onset of oxidation and allowing the generation of a higher polaron density. For example, for P3HT the $V_{O N}$ value decreases to $0.05 \mathrm{~V}$, the $\mathrm{ON}$ current increases by $\sim 3$ times, and the transconductance $\left(g_{\mathrm{m}}\right)$ increases to $4 \mathrm{mS}$, which is, to the best of our knowledge, the highest transconductance of P3HT OECT reported. These results demonstrate a simple, but effective way of using a small molecule additive, such as DBSA, and a possibility to utilise otherwise unsuitable polymers with deep highest occupied molecular orbital (HOMO) levels, for low-power accumulation mode OECTs.
\end{abstract}

\section{Introduction}

Organic electrochemical devices (OEDs) utilise conducting and semiconducting polymers to engineer devices operating with high current densities and low power. OEDs have many applications which include biosensing, ${ }^{1-6}$ electrochemical energy storage, ${ }^{7-12}$ and neuromorphics. ${ }^{13,14}$ The organic electrochemical transistor (OECT) is one of the most widely deployed OEDs and consists of a channel of conjugated polymer contacted by source and drain metal electrodes. ${ }^{15}$ The polymer channel is submerged in an electrolyte and a gating electrode is exposed to the electrolyte but does not contact the channel directly. ${ }^{16}$

\footnotetext{
${ }^{a}$ Department of Physics and Centre for Processable Electronics,

Imperial College London, South Kensington Campus, London, SW7 2AZ, UK. E-mail: ji-seon.kim@imperial.ac.uk

${ }^{b}$ Department of Chemical Engineering and Biotechnology, University of Cambridge, Cambridge, CB3 OAS, UK

$\dagger$ Electronic supplementary information (ESI) available: Transfer curves with square root of $I_{\mathrm{DS}}$ to estimate the $V_{\mathrm{ON}}$ values; UV-vis absorbance, air photoelectron spectroscopy and kelvin probe measurements of pristine and chemically doped P3HT; ERRS measurements demonstrating the volumetric effect. See DOI: $10.1039 /$ dotc02149k
}

This allows a gate bias induced ion-redistribution which modulates the conductance of the polymer channel. The electrolyte is selected such that the polymer layer swells and allows ionic penetration into the bulk of the active channel upon application of a gate bias. ${ }^{17}$ The high concentration of ions within the polymer channel is balanced by counter charge carriers that are injected into the polymer thus causing bulk electrochemical doping/dedoping. ${ }^{18}$ This generates volumetric charging in the channel, which leads to high transconductance values $\left(\delta I_{\mathrm{DS}}\right)$ $\delta V_{\mathrm{DS}}$ ); a figure of merit for OECTs. ${ }^{16}$

The most common material used for OECTs is PEDOT:PSS, which consists of a mixture of two ionomers: negatively charged polystyrene sulphonate, PSS $^{-}$blended with poly(3,4-ethylenedioxythiophene), PEDOT. PEDOT is p-type doped by the deprotonated PSS $^{-}$and contains a typical hole concentration of $\sim 3 \times$ $10^{20}$ holes $\mathrm{cm}^{-3}$ making it highly conductive $\left(0.2-2100 \mathrm{~S} \mathrm{~cm}^{-1}\right),{ }^{19,20}$ thus the OECT operates in depletion mode whereby it is normally ON under no external bias application. It therefore typically requires a gate bias of approximately $-0.7 \mathrm{~V}$ to switch it OFF, consuming power in its OFF state. ${ }^{15}$ Recently, semiconducting polymers, that are typically insulating in their pristine state, have been employed to develop accumulation mode OECT devices. ${ }^{16,21-23}$ These polymers 
are normally in an OFF state until electrochemically doped and switched ON thus consuming less power. Additionally, in biosensing they provide increased signal of detection in the presence of an analyte compared to depletion mode OECTs.

Semiconducting polymers swell in typical organic solvents allowing ion penetration and high transconductance. ${ }^{17} \mathrm{How}^{-}$ ever, for biosensing applications it is essential to operate in aqueous environments. ${ }^{15,24}$ Due to the non-polar nature of polymers, this limits their ability to swell and thus limits performance in aqueous media. A biphasic electrolyte platform developed by Duong et al. incorporates two immiscible electrolytes, an organic layer in direct contact with the polymer and aqueous layer on top. ${ }^{25}$ The former is selected because it allows ion penetration into P3HT so it can operate as an OECT, whilst the less dense aqueous layer interfaces with the biological environment of interest. This development opens the potential to utilise a wide range of organic semiconducting polymers that have been previously developed over the past few decades for transistor and solar cell applications.

In order to implement organic semiconducting polymers in OECTs for biosensing applications, particularly with live cells or cell membranes, it is essential to operate the device at lower voltages $(<1 \mathrm{~V})$ in order not to adversely affect the functionality and structure of the biological systems. ${ }^{26-28}$ Low-voltage operation is also of key interest particularly important for use with low supply voltage thin-film batteries and portable devices. ${ }^{29}$ The operation voltage of the device utilising p-type doping/ dedoping processes is partially governed by the highest occupied molecular orbital (HOMO) level of the polymer. ${ }^{25}$ Many polymers with deep HOMO levels are not compatible for biosensing applications (as the operation voltage would be too high when typical metal electrodes such as $\mathrm{Au}$ or $\mathrm{Ag}$ are used) despite showing promising electronic properties and high charge carrier mobility in OFET devices. In this study we report a simple but effective method for reducing the OECT operating voltage by using a small molecule additive, dodecylbenzenesulfonate (DBSA) that can be added directly to the electrolyte. Previously, DBSA has been added to PEDOT:PSS formulations to act as a surfactant to improve thin film processing. ${ }^{30}$ Herein, we demonstrate how DBSA can effectively reduce operating-voltages, enhance performance via increasing the ON current and transconductance, and lower the power consumption of the OECT device when added to the electrolyte. By using a range of p-type polymers, we confirm that this method can be utilised in organic semiconducting polymers with deep HOMO levels for high performance OECTs with operating voltages low enough for biosensing applications.

We first demonstrate the benefit of adding DBSA (Fig. 1) to the electrolyte (tetrabutylammonium hexafluorophosphate, TBA $\mathrm{PF}_{6}$ ) in a $1: 1$ ratio to enhance OECT performance consisting of P3HT polymer channels. We reveal that adding DBSA increases the ON current and transconductance and decreases the operation voltage resulting in significant enhancement in device performance. We demonstrate this further by applying the DBSA additive to OECTs fabricated with other conjugated polymers including DPPT-TT and PBTTT. We select PBTTT because it has been shown to be a high performance material for use in OECTs. ${ }^{25}$ DPPT-TT, on the other hand, has yet to be reported in the literature for use in OECTs, which led us to investigate whether its well-known high performance in OFETs translates to high performance in OECTs. ${ }^{30-32}$ We observe the same effects of adding DBSA to the gating electrolyte for both DPPT-TT and PBTTT as for P3HT.

To elucidate the effects of DBSA and the electrolyte mixture (containing DBSA), we use electrochemical resonant Raman spectroscopy (ERRS), which provides a powerful tool to probe changes to the vibrational modes during polaron (charge) formation in polymers. Polarons are formed in conjugated polymers when the polymer becomes charged. Owing to the strong electron-phonon coupling in conjugated polymers, the charge is accompanied by a distortion of the equilibrium position of the local nuclei resulting in changes to the vibrational modes (so called lattice relaxation). ERRS generates polarons in the polymer via the application of an oxidising potential (electrochemically doping) in a three-electrode electrochemical cell, whilst probing changes of the vibrational modes of the polymer in situ using resonant Raman spectroscopy. ${ }^{33}$

We find that the addition of DBSA acts to generate polarons at a lower potential and to a higher density. Ultimately this decreases the operating-voltage of the OECT and paves the way to implementing other high-performance p-type polymers with deeper HOMO levels as channel materials for OECTs for a variety of applications, including biosensing.

\section{Experimental}

\section{Materials}

Details of the polymers used in this work are as follows: RRP3HT (95.2\% regioregularity) was bought from Merck, with weight-average molecular weight $\left(M_{\mathrm{w}}\right) \sim 36.6 \mathrm{~kg} \mathrm{~mol}^{-1}$ and polydispersity index of 2; PBTTT-C ${ }_{14}$ was purchased from Sigma Aldrich, with weight-average molecular weight $\left(M_{\mathrm{w}}\right) 12 \mathrm{kDa}$; DPPT-TT- $\mathrm{C}_{10} \mathrm{C}_{8}$ was purchased from 1-material (1M) with weight-average molecular weight $\left(M_{\mathrm{w}}\right) 87$.

\section{Methods}

Film preparation. Polymer was dissolved in chlorobenzene at a concentration of $20 \mathrm{mg} \mathrm{ml}^{-1}$ and left stirring at $80{ }^{\circ} \mathrm{C}$ overnight. Fluorine-doped tin oxide (FTO) coated glass substrates were cleaned in a sonicator for 10 minutes at a time in soap solution, followed by acetone and finally IPA before plasma ashing. The solution was deposited on the clean FTO glass substrates and spun at $1000 \mathrm{rpm}$ for 60 seconds. Films were then dried under vacuum overnight.

Electrochemical measurements. All electrochemical measurements were made using an Autolab potentiostat (PGSTAT) and the custom-made three electrode electrochemical cell. The cells components are as follows: thin film deposited on FTO coated glass (working electrode), platinum dispersed on FTO coated glass (counter electrode), silver (Ag) wire (pseudo 
RR-P3HT

a)

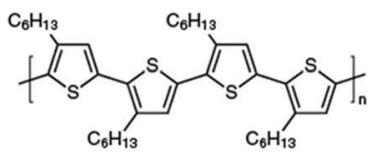

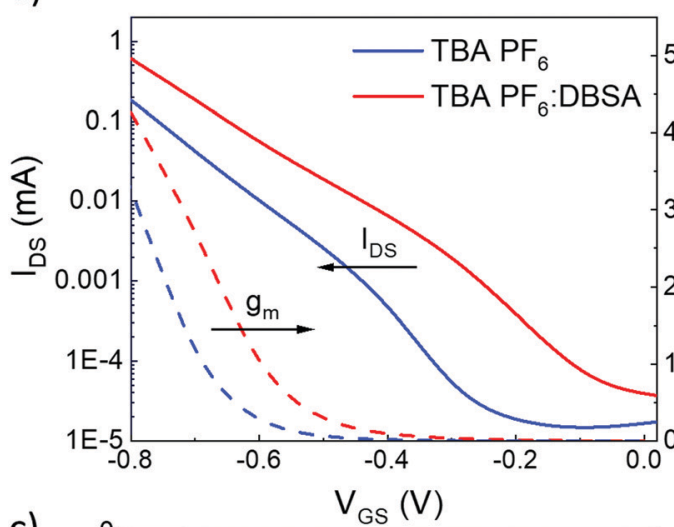

c)

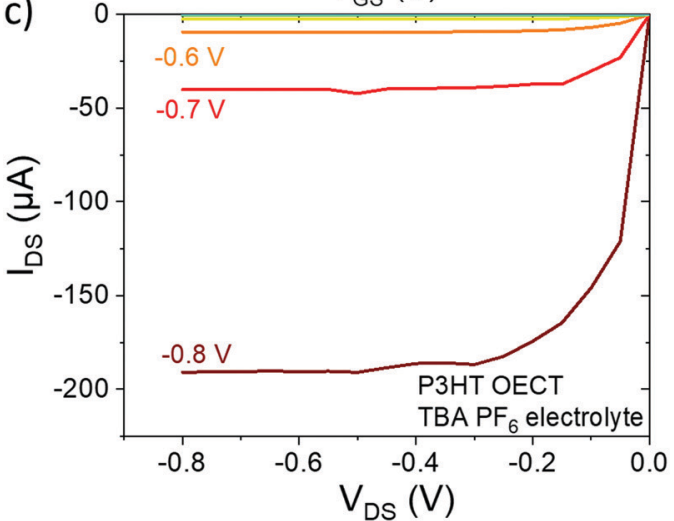

TBA PF $_{6}$<smiles>CCCC[N+](CCCC)(CCCC)CCCC</smiles>

b)
DBSA<smiles>CCCCc1ccc(S(=O)(=O)O)cc1</smiles>

reference electrode), and 0.1 $\mathrm{M} \mathrm{tBuNH} \mathrm{PF}_{6}$ in acetonitrile (inert electrolyte). Cyclic voltammetry was measured by applying an oxidising potential to the working electrode and scanning the potential from $-0.5 \mathrm{~V}$ to $1.2 \mathrm{~V} v \mathrm{~s}$. Ag at a scan rate of $20 \mathrm{mV} \mathrm{s}^{-1}$, followed by reversing the direction. The current response waveform was measured simultaneously. Chronoamperometry was used to oxidise the polymer film during in situ spectroscopy measurements. It was performed by applying an excitation square-wave potential to the working electrode in the following configuration: $0 \mathrm{~V}$ for 20 seconds (neutral state), an oxidising potential $\left(V_{\mathrm{ox}}\right)$ for 40 seconds (oxidised state), and $0 \mathrm{~V}$ for 20 seconds (back to neutral state). In situ Raman and UV-vis measurements were taken during the application of an oxidising potential when the current response reached steady state (usually after 5-10 seconds).

In situ electrochemical resonant Raman spectroscopy. Five excitation wavelengths were used; 457, 488, and $514 \mathrm{~nm} \mathrm{(11,} \mathrm{9,}$ and $2 \mathrm{~mW}$ ), He-Ne laser (Renishaw RL633) emission at $633 \mathrm{~nm}$ (12 mW), diode laser (Renishaw HPNIR785) with emission at $785 \mathrm{~nm}(130 \mathrm{~mW})$. Access to a Nd:YAG laser with emission at $1064 \mathrm{~nm}$ was provided by Renishaw. These measurements were taken on an in-house inVia microscope with an InGaAs detector. $10 \%$ power and $25 \%$ defocus were applied to avoid photodegradation of the film.

OECT device fabrication. OECT devices were oxygen plasma ashed before spin-coating the polymer solutions at $1000 \mathrm{rpm}$ for $60 \mathrm{~s}$. This was followed by baking the devices at $60{ }^{\circ} \mathrm{C}$ for $60 \mathrm{~s}$ before removing the sacrificial parylene layer. Following the parylene peel-off the devices were further baked at $140{ }^{\circ} \mathrm{C}$ for $30 \mathrm{~min}$. A polydimethylsiloxane (PDMS) well was fabricated using Sylgard-184 base and a curing agent in a 10:1 ratio. The PDMS well contained the electrolyte with a total volume of $300 \mu \mathrm{l}$. TBA PF6 electrolytes and TBA $\mathrm{PF}_{6}$ : DBSA mixed electrolytes (in both ACN and DCM) had a concentration of $0.1 \mathrm{M}$. The ratio of $\mathrm{TBA}_{\mathrm{PF}}$ : DBSA was kept constant at $1: 1$ for all mixed electrolyte measurements.

Device characterization. Transfer and output characteristics were measured using a Keysight B2902A precision source/ measure unit.

Energetics. Ambient photoemission measurements were taken using an APS04 Ambient Photoemission system (KP Technology). A $2 \mathrm{~mm}$ gold tip was used under atmospheric conditions. 
Samples were deposited on ITO to allow grounding of the organic thin film. Multiple positions were measured and data processed following the method reported by Baike et al. ${ }^{34}$

\section{Results and discussion}

First, we demonstrate the effect of DBSA as a small molecule electrolyte additive, which enhances the electrochemical doping mechanism and device characteristics of OECTs fabricated from conjugated polymers. We start with P3HT and extend the application to PBTTT and DPPT-TT.

Fig. 1 shows the transfer characteristics of OECTs fabricated with P3HT as a channel material and TBA $\mathrm{PF}_{6}$ (blue) and TBA $\mathrm{PF}_{6}$ : DBSA $(1: 1)$ (red) in acetonitrile (ACN) as a gating electrolyte. With $\mathrm{TBA}_{\mathrm{PF}}$ the $\mathrm{ON}$ currents of the device are $0.04 \mathrm{~mA}$ at a gate bias of $-0.7 \mathrm{~V}$. The ON/OFF ratio is $\sim 10^{4}$ with an OFF current $\sim 2 \times 10^{-5} \mathrm{~mA}$. When DBSA is added to the TBA $\mathrm{PF}_{6}$ gating electrolyte the ON current increases by over 4 times to $0.18 \mathrm{~mA}$ with no significant change in the ON/OFF ratio $\left(\sim 10^{4}\right)$ and an OFF current of $\sim 4 \times 10^{-5} \mathrm{~mA}$. The maximum (measured) transconductance, $g_{\mathrm{m}}$ (at $V_{\mathrm{GS}}=-0.8 \mathrm{~V}$ ), increases from $3 \mathrm{mS}$ (no DBSA) to $4 \mathrm{mS}$ (with DBSA). The sub $V_{\text {ON }}$ characteristics with both electrolytes are flat and show sharp turn-on. Most notably DBSA causes a significant decrease in the operation voltage with a drop in $V_{\mathrm{ON}}$ value from $\sim 0.70 \mathrm{~V}$ to $\sim 0.58 \mathrm{~V}\left(\Delta V_{\mathrm{ON}}=0.12 \mathrm{~V}\right)$ (Fig. $\mathrm{S} 1$ for $I_{\mathrm{DS}}{ }^{1 / 2}$ plots, ESI $\left.\dagger\right)$. Fig. 1b shows the transient gate bias pulse measurements $\left(V_{\mathrm{GS}}=-0.6 \mathrm{~V}, V_{\mathrm{DS}}=-0.6 \mathrm{~V}\right)$. During a gate bias of $-0.6 \mathrm{~V}$ (in TBA $\mathrm{PF}_{6}$ electrolyte) there is a drain current of $\sim 0.4 \mathrm{~mA}$. Adding DBSA causes a large amplification of the $I_{\mathrm{DS}}$ by $\sim 3 \times$ (from $0.4 \mathrm{~mA}$ to $1.2 \mathrm{~mA}$ ), whilst the transient turn-on/ turn-off characteristics remain fast. Adding DBSA also has a positive effect on the output characteristics of P3HT (Fig. 1c and d): $I_{\mathrm{DS}}$ increases $\sim 3$ times from $\sim-190 \mu \mathrm{A}$ without DBSA to $\sim-600 \mathrm{~mA}$ with DBSA $\left(V_{\mathrm{GS}}=-0.8 \mathrm{~V}\right)$.

The state-of-the art P3HT OECT device performance reported so far achieves an ON current of $0.3 \mathrm{~mA}, V_{\mathrm{ON}}$ of $-0.2 \mathrm{~V}$, and a transconductance of $2 \mathrm{mS} .{ }^{23}$ We show that the $V_{\mathrm{ON}}$ value can be decreased to $0.05 \mathrm{~V}$, the ON current increased by $\sim 3$ times, and the transconductance, $g_{\mathrm{m}}$ increased from $3 \mathrm{mS}$ to $4 \mathrm{mS}$. To the best of our knowledge this is the highest transconductance of a P3HT OECT reported, with the next highest being $2 \mathrm{mS}^{23}$

The addition of DBSA appears to improve device characteristics by lowering the operation voltage and increasing the drain current, thus lowering the power consumption of the device. This indicates the ability of DBSA to improve the ion-induced modulation of the polymer channel conductance. This correlates with a decrease in the oxidation onset (from $0.60 \mathrm{~V}$ to $0.29 \mathrm{~V}$ vs. Ag) of P3HT thin films when DBSA is present in the electrolyte mixture (CV Fig. 2). The two oxidation peaks of P3HT in $\mathrm{TBA} \mathrm{PF}_{6}$ electrolyte (green curve) (Fig. 2a) have previously been assigned to the oxidation of crystalline $(0.6-0.8 \mathrm{~V})$ and amorphous (1.0-1.2 V) phases. ${ }^{33,35,36}$ The relative ratio amplitude of the crystalline and amorphous oxidation peaks ( $I_{\text {crystalline }}: I_{\text {amorphous }}$ ) is $0.27: 1$ in $\mathrm{TBA} \mathrm{PF}_{6}$ electrolyte and remains approximately the same when DBSA is added $(0.26: 1)$. This indicates that both crystalline and amorphous polymer a)

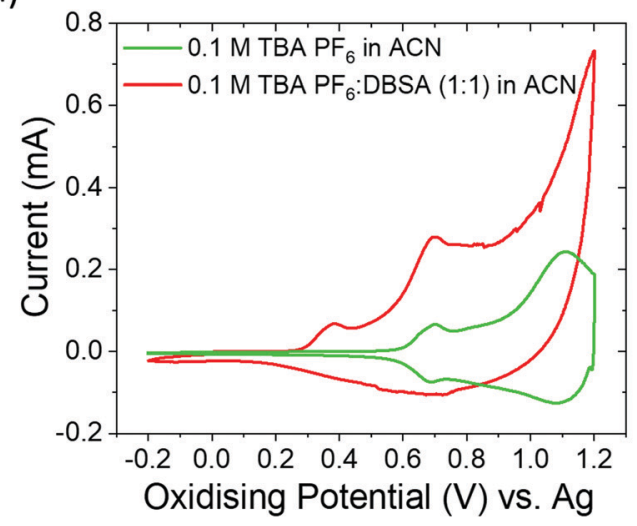

b)

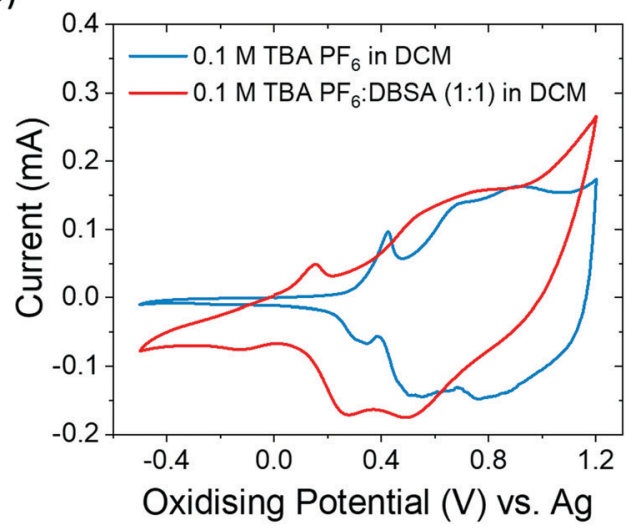

c)

\begin{tabular}{|c|c|c|}
\hline Electrolyte mixture & $\begin{array}{c}\text { Oxidation Onset, Vox (V } \\
\text { vs. } \mathbf{A g})\end{array}$ & $\begin{array}{c}\text { Shift of Vox with DBSA } \\
\text { added (V vs. Ag) }\end{array}$ \\
\hline $0.1 \mathrm{M} \mathrm{TBA} \mathrm{PF}_{6}$ in ACN & 0.60 & -0.31 \\
\cline { 1 - 2 } $0.1 \mathrm{M} \mathrm{TBA} \mathrm{PF}$ :DBSA (1:1) in ACN & 0.29 & \multirow{2}{*}{-0.28} \\
\hline $0.1 \mathrm{M} \mathrm{TBA} \mathrm{PF}_{6}$ in DCM & 0.32 & \\
\hline $0.1 \mathrm{M} \mathrm{TBA} \mathrm{PF}_{6}: \mathrm{DBSA}_{(1: 1)}$ in DCM & 0.04 & \\
\hline
\end{tabular}

Fig. 2 Cyclic voltammograms of P3HT thin films in different electrolytes. (a) TBA PF 6 (green) and TBA PF 6 : DBSA (1:1) mixture (red) in acetonitrile (ACN). (b) $\mathrm{TBA} \mathrm{PF}_{6}$ (blue) and TBA PF 6 : DBSA (1:1) mixture (red) in dichloromethane (DCM). (c) Table summarising the oxidation onsets in each electrolyte mixture. 
phases sufficiently swell and allow ion penetration to an equal extent in both electrolytes suggesting that DBSA does not alter the interactions between the solvent and different polymer phases.

It is common to use dichloromethane (DCM) as an electrolyte solvent for biosensing applications. ${ }^{23}$ Similarly to ACN, DCM can sufficiently dissolve salts, and it can efficiently swell polymer films without dissolving individual chains. DCM, however, is far less polar than ACN and is therefore largely immiscible with water facilitating an interface with biological environments and media. ${ }^{25}$

Interestingly, the oxidation onset (using $\mathrm{TBA}^{\mathrm{PF}_{6}}$ salt only) occurs at a lower potential in DCM compared to ACN. This is due to the higher polarity of ACN which may have two possible effects leading to different oxidation onsets measured for the polymer. Firstly, there is a higher relative energy distance (RED) between P3HT and ACN compared to P3HT and DCM, which makes DCM a better solvent causing the polymer film to swell more (without fully dissolving the film). Greater swelling of the polymer is expected to reduce resistance to ion penetration. This has been previously calculated for PBTTT using the Hansen solubility parameter space where the RED for DCM is $<1$ (0.64) and ACN > 1 (2.6). ${ }^{25,37}$ We calculate the RED between P3HT and DCM (1.01) and between P3HT and ACN (2.76) using solubility parameters reported by Zhang et al. ${ }^{38}$ Secondly, the higher polarity of ACN will more strongly solvate the electrolyte ions, which can cause an overpotential in the electrochemical oxidation of the polymer film. ${ }^{22}$ The primary effect of DBSA in both solvents is a significant reduction in the oxidation onset of the polymer film.

To elucidate the doping mechanism of the admixture of DBSA and TBA $\mathrm{PF}_{6}$ electrolyte we employ ERRS to directly probe polaron formation and associated conformation changes of the polymer in situ. Using ERRS we can distinguish between primary and secondary doping effects of DBSA. Fig. 3a shows the ERRS spectra of $\mathrm{P} 3 \mathrm{HT}$ in $\mathrm{TBA} \mathrm{PF}_{6}$ electrolyte during a series of oxidising potential applications. The most intense peak $\left(1448 \mathrm{~cm}^{-1}\right)$ is assigned to the intra-ring $\mathrm{C}=\mathrm{C}$ bond vibrational mode and the weaker peak $\left(1382 \mathrm{~cm}^{-1}\right)$ is assigned to the intraring $\mathrm{C}-\mathrm{C}$ bond vibrational mode. ${ }^{39}$ The ERRS spectra as a function of applied oxidising potential displays the characteristic Raman signatures of hole polarons forming in different polymer phases; holes form first in the crystalline phase at lower potentials $(\sim 0.6 \mathrm{~V})$ followed by the amorphous phase at higher potentials $(\sim 1.0 \mathrm{~V})$. These spectral changes we observe during $V_{\text {ox }}$ provide information on conformational changes of the P3HT during polaron formation. ${ }^{33}$ Most notably is the downshift of the $\mathrm{C}=\mathrm{C}$ peak to lower wavenumber (from 1448 to $1404 \mathrm{~cm}^{-1}$ ) and an increase in the relative intensity of the $\mathrm{C}-\mathrm{C} / \mathrm{C}=\mathrm{C}$ peaks (from 0.38 to 0.93 ), indicating that the $\mathrm{C}=\mathrm{C}$ bond force constant weakens and the polymer adopts a more planar conformation during polaron formation. After discharging the film $(0 \mathrm{~V})$ to its neutral state, we observe the Raman spectra revert back to its original shape and position (Fig. 3b), which indicates the conformational reorganisation during polaron formation is entirely reversible.
When a low concentration of DBSA $(0.05 \mathrm{M})$ is added to the electrolyte mixture (TBA $\mathrm{PF}_{6}$ :DBSA $(1: 1)$ ) similar spectral changes occur during polaron formation (Fig. 3c). Monitoring the $\mathrm{C}=\mathrm{C}$ peak position as a function of oxidising potential (Fig. 3d) reveals that the conversion of neutral polymer to charged polymer has already occurred at $0.4 \mathrm{~V}$ when DBSA is present (red line) compared to $0.6 \mathrm{~V}$ when DBSA is absent (blue line). The formation of hole polarons at a lower oxidising potential correlates with the lower operation voltage in the OECT when DBSA is added to the gating electrolyte. Furthermore, when DBSA is added to the electrolyte we measure a higher polaron density generated in the film $\left(1.79 \times 10^{21}\right.$ holes $\mathrm{cm}^{-3}$ at $1.0 \mathrm{~V})$ compared to just TBA $\mathrm{PF}_{6}\left(1.16 \times 10^{21}\right.$ holes $\mathrm{cm}^{-3}$ at $\left.1.0 \mathrm{~V}\right)$ (Fig. 3e). The method used for calculating polaron density has been previously reported. ${ }^{33}$ The polaron density generated in this study is typical of the polaron density generated in OECT devices. $^{22}$ This correlates well with the higher drain current measured in the OECT device when DBSA is added. Thus, we confirm that DBSA enhances the ion-induced modulation of the device by altering the primary electrochemical doping of the polymer.

We are able to clarify that the electrochemical doping of the polymer is still predominantly caused by the formation of ion pairs between charged polymer and $\mathrm{PF}_{6}{ }^{-}$anions, as DBSA itself causes irreversible doping of the polymer (Fig. 4). Fig. 4a shows the behaviour of DBSA in an electrochemical cell with no other salts present by exposing a P3HT thin film to 0.1 M DBSA in ACN electrolyte and measuring in situ ERRS. We observe the typical hole polaron signature in $\mathrm{P} 3 \mathrm{HT}$ even at $0 \mathrm{~V}$, indicating the ability of DBSA to chemically dope the polymer film when no other salts are present. We monitor the $\mathrm{C}=\mathrm{C}$ peak position as a function of oxidising potential and find the doping level has a minor dependence on applied bias indicated by the $\mathrm{C}=\mathrm{C}$ peak downshifting from $1425 \mathrm{~cm}^{-1}$ to $1417 \mathrm{~cm}^{-1}$ (Fig. 4b); the electric field causes more DBSA anions (dissociated from protons) to drift and penetrate into the P3HT film causing further doping. After discharge $(0 \mathrm{~V})$ the film is irreversibly doped and the $\mathrm{C}=\mathrm{C}$ peak position remains at $1417 \mathrm{~cm}^{-1}$ (green triangle Fig. 4a). Further evidence for irreversible p-type doping caused by DBSA is provided by changes in the UV-Vis absorbance spectra, HOMO level, and work function (see Fig. S2, ESI $\dagger$ ). Thus, we elucidate that the mixed ionic electrolyte (with DBSA and TBA: $\mathrm{PF}_{6}$ ) causes reversible electrochemical doping of the polymer caused by the formation of ion pairs between charged polymer and $\mathrm{PF}_{6}{ }^{-}$anions. This suggests that the effect of DBSA in the mixed electrolyte is to modify the interaction between the polymer and the electrolyte allowing electrochemical doping at a lower voltage. DBSA has previously been used as a surfactant added to PEDOT:PSS formulations to improve wettability. ${ }^{30} \mathrm{We}$ believe that DBSA could be having a similar effect on P3HT, interacting with the polymer and acting as a surfactant to enhance the strength of the interactions between the polymer and the electrolyte. In other words, DBSA may modulate the RED between the polymer and the solvent. We demonstrated the effect of RED between the solvent and the polymer on oxidation onset during cyclic voltammetry measurements: 
a)

c)
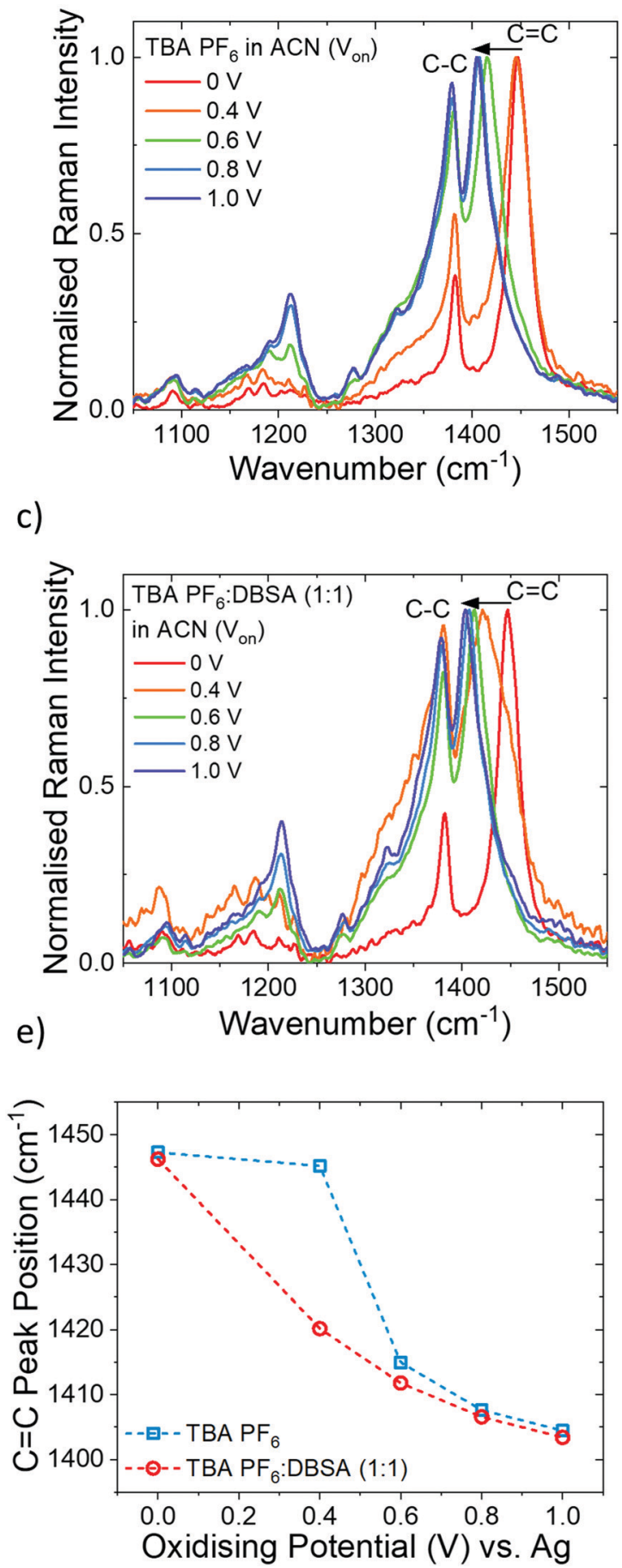

b)

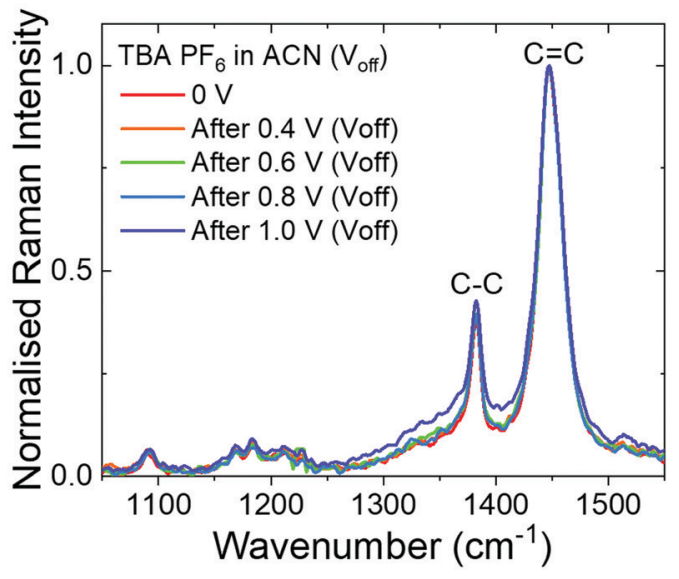

d)

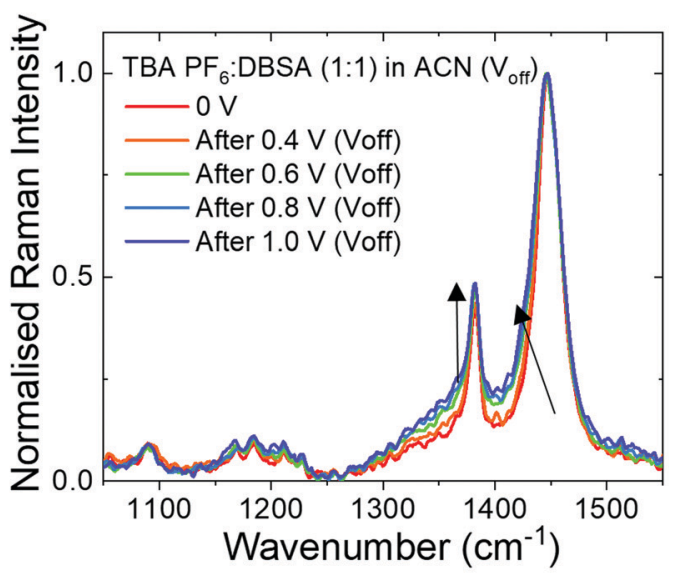

(1)

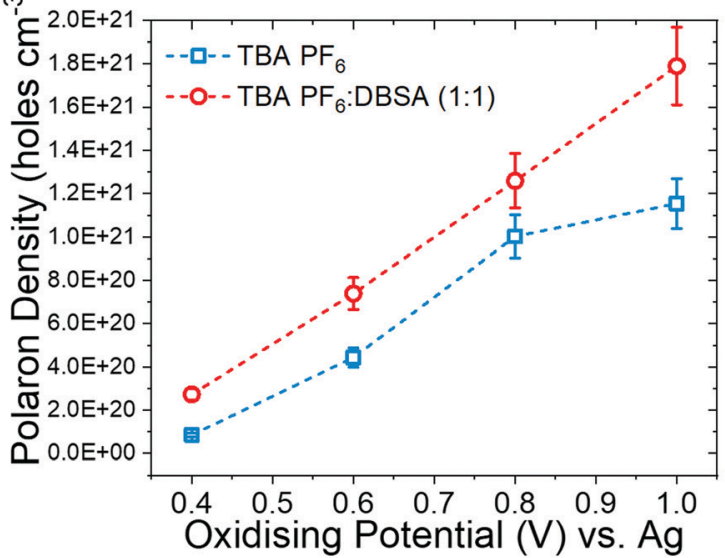

Fig. 3 Characterising doping mechanism of $\mathrm{TBA} \mathrm{PF}_{6}$ and $\mathrm{TBA}_{\mathrm{PF}} / \mathrm{DBSA}$ electrolytes: reversible electrochemical doping of $\mathrm{TBA}^{\mathrm{P}} \mathrm{PF}_{6} / \mathrm{DBSA}$ electrolyte mixtures. (a)-(d) in situ ERRS measurements with different electrolyte mixtures: $0.1 \mathrm{M} \mathrm{TBA} \mathrm{PF}_{6}$ in acetonitrile, $V_{\mathrm{ON}}$ and $V_{\text {off }}(\mathrm{a})$ and (b); $0.1 \mathrm{M}$ TBA PF 6 : DBSA (1:1) in acetonitrile, $V_{O N}$ and $V_{\text {off }}$ (c) and (d). (e) Peak position of the peak assigned to the $\mathrm{C}=\mathrm{C}$ vibrational mode as a function of oxidising potential (V). (f) Polaron density generated in the P3HT thin film as a function of oxidising potential $(V)$ with $\mathrm{TBA}^{\mathrm{PF}} 6$ (blue) and with DBSA (red) added to the electrolyte mixture.

there is a lower RED between DCM and P3HT compared to ACN and $\mathrm{P} 3 \mathrm{HT}$, which results in a reduction in the oxidation onset of $0.28 \mathrm{~V}$ (Fig. 2). Thus, the RED between the polymer and the electrolyte solvent appears to influence the injection barrier into the film. By adding DBSA to the electrolyte, it may reduce the RED, thus causing a higher mass in the polymer film (solvent and ions), ultimately decreasing the injection barrier into the film.

Lastly, we note that the ionic strength of the mixed DBSA: TBA $\mathrm{PF}_{6}$ electrolyte is the same as the neat $\mathrm{TBA} \mathrm{PF}_{6}$ electrolyte. 
Ionic strength of a solution, $I$, is dependent on the concentration of ions in the solution: $I=1 / 2 \Sigma c_{\mathrm{i}} z_{\mathrm{i}}{ }^{2}$, where $c_{\mathrm{i}}$ is the molar concentration of all ions in the solution, and $z_{\mathrm{i}}$ is the charge number of the ion. Since we control the molar concentration to be the same for both the mixed and neat electrolyte ( $0.1 \mathrm{M}$ for both), and the charge number of all ions is parity $\left(\mathrm{TBA}^{+} v s . \mathrm{H}^{+}\right.$and $\mathrm{PF}_{6}{ }^{-}$vs. $\mathrm{DSBA}^{-}$), we are thus able to rule out the possibility of ionic strength causing the effect of DBSA observed in lowering the oxidation onset of the polymer thin films.

After discharging the film $(0 \mathrm{~V})$ exposed to the mixed electrolyte ( $\mathrm{TBA} \mathrm{PF}_{6}$ :DBSA) the Raman signatures revert to that of the original, neutral polymer, revealing no change in polymer conformation or molecular morphology, and thus ruling out any secondary doping effect of DBSA (Fig. 3d). There is, however, a low intensity polaron signature evident from the slight broadening of the $\mathrm{C}=\mathrm{C}$ peak to lower wavenumber and a slight increase in $\mathrm{C}-\mathrm{C}$ relative peak intensity (indicated by arrows in Fig. 3d). This reveals that the doping process isn't completely reversible (at $0.6 \mathrm{~V}$ and above) and leads to a low density of polarons remaining in the film. This correlates with the very low level drain current during no gate bias $(0 \mathrm{~V})$ in the transient gate bias pulse measurements (Fig. 1b), which wasn't observed before DBSA was added.

Currently there is much debate within the field of organic mixed ionic-electronic devices about the extent of bulk doping (volumetric effect) of organic conjugated polymers with alkyl side chains using common organic electrolytes. Using ERRS we confirm the volumetric effect in polymer thin films (Fig. S3, ESI $\dagger$ ). We compare the strength of the Raman signals from films of different thickness (124 nm vs. $270 \mathrm{~nm})$ with controlled laser focus (micron-size depth resolution) for each measurement. We observe a signal twice as strong for the thicker film $(240 \mathrm{~nm})$, which is just over twice the thickness of the $124 \mathrm{~nm}$ film. The signal strength is governed by the Raman scattering cross section, which in turn is dependent upon the number of Raman active species present. ${ }^{39} 785 \mathrm{~nm}$ excitation is resonant with the polaron absorption and thus a higher intensity indicates a greater number of polarons generated in the film (due to its greater thickness and bulk doping effect). This is in close agreement with our measurement of the number of charges extracted after the oxidising pulse for which twice as many were extracted from the thicker film $\left(4.7 \times 10^{16}\right.$ vs. $2.3 \times 10^{16}$ holes $)$. Our results support the notion that bulk electrochemical doping occurs in organic conjugated polymers even with alkyl side chains using acetonitrile-based electrolytes.

Now we show the beneficial effects of DBSA addition to other conjugated polymer OECTs including DPPT-TT and PBTTT devices, which suggests its effect can be extended and applied to other conjugated polymer systems. Fig. 5a shows the transfer curve characteristics of OECTs fabricated with DPPT-TT and gating electrolytes comprising TBA $\mathrm{PF}_{6}$ (black) and TBA $\mathrm{PF}_{6}$ : DBSA $(1: 1)$ (red) in ACN. With the TBA $\mathrm{PF}_{6}$ electrolyte the ON current of the device is $\sim-1.0 \mu \mathrm{A}$ at a gate bias of $-0.7 \mathrm{~V}$. The ON/OFF ratio is $\sim 10^{4}$ with an OFF current of $\sim-10^{-4} \mu \mathrm{A}$. When DBSA is added to the gating electrolyte the ON current a)

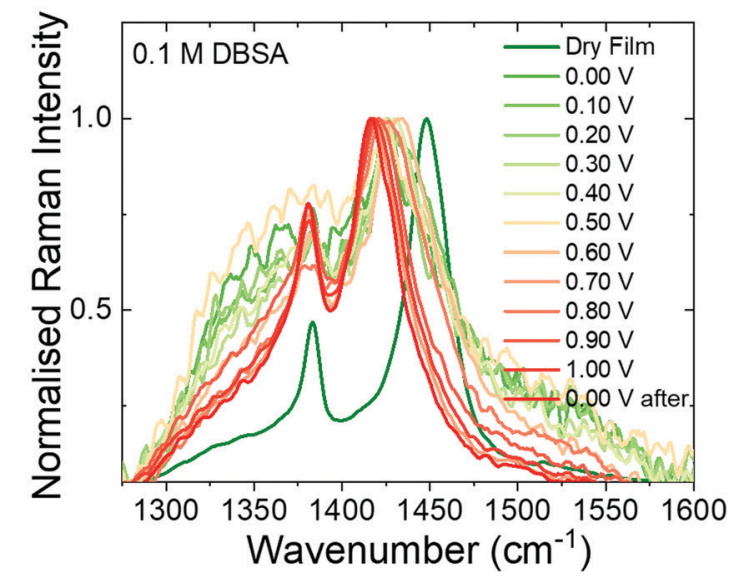

b)

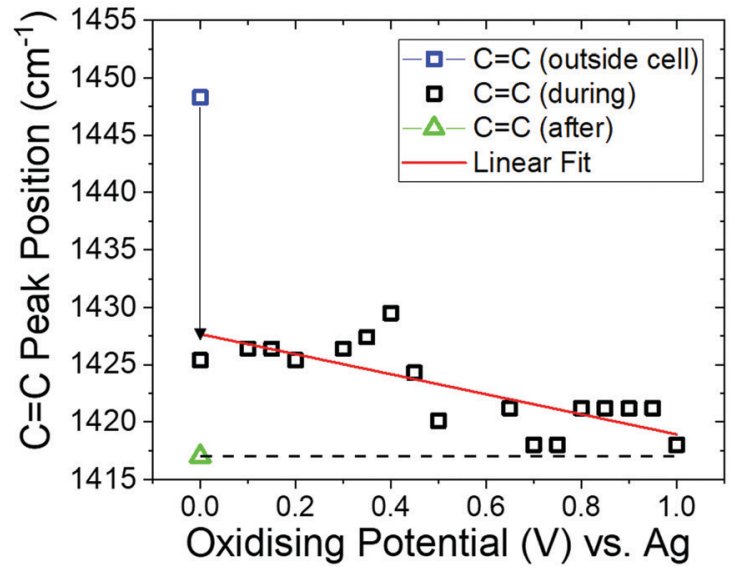

Fig. 4 Irreversible chemical doping of DBSA in ACN electrolyte. (a) ERRS spectra of P3HT doped using DBSA in ACN electrolyte. (b) Peak position of the $\mathrm{C}=\mathrm{C}$ vibrational mode as a function of oxidising potential (V).

increases to $\sim-10.0 \mu \mathrm{A}$, and whilst the OFF current also increases the ON/OFF ratio remains $\sim 10^{4}$. Most remarkable is the significant decrease in the operation voltage upon addition of DBSA with a drop in $V_{\mathrm{ON}}$ value from $\sim 0.63 \mathrm{~V}$ to $\sim 0.48 \mathrm{~V}$ $\left(\Delta V_{\mathrm{ON}}=-0.15 \mathrm{~V}\right)$ (Fig. S1 for $I_{\mathrm{DS}}^{1 / 2}$ plots, ESI $\left.\dagger\right)$. Fig. $5 \mathrm{~b}$ shows the transient gate bias pulse measurements. During a gate bias of

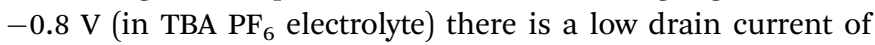
$\sim-0.8 \mu \mathrm{A}$. Adding DBSA causes a large amplification of the $I_{\mathrm{DS}}$ by $\sim 18 \times$ (from $0.8 \mu \mathrm{A}$ to $14.0 \mu \mathrm{A}$ ), whilst the transient turn-on/ turn-off characteristics remain fast. Albeit to a lesser extent the output characteristics are also improved upon DBSA addition: $I_{\mathrm{DS}}$ increases $\sim 4$ times from $-1 \mu \mathrm{A}$ to $-4 \mu \mathrm{A}$ (at $V_{\mathrm{GS}}=0.7 \mathrm{~V}$ ) (Fig. 5c and d).

We report, for the first time, that DPPT-TT is a lower performance channel material compared to P3HT and PBTTT. For DPPT-TT, the ON current $(\sim-1.0 \mu \mathrm{A}), V_{\text {ON }}$ value $(\sim 0.63 \mathrm{~V})$, and transconductance $(0.012 \mathrm{mS})$, are far below the performance of P3HT and PBTTT. Still, the addition of DBSA to the electrolyte is effective in enhancing its performance and results in a significant increase in the ON current and decrease in the operation voltage. The positive effect of adding DBSA to the electrolyte extends not 


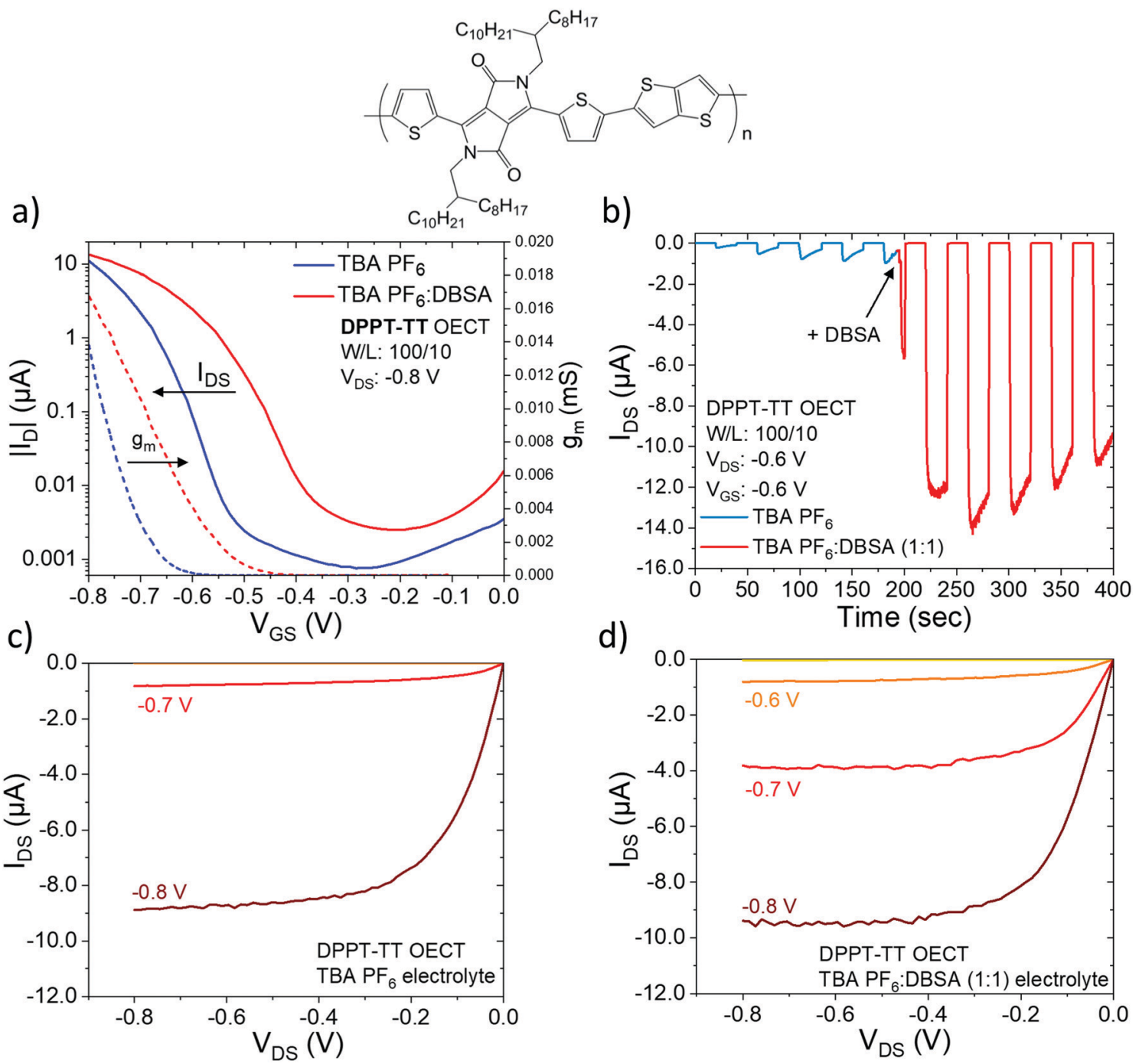

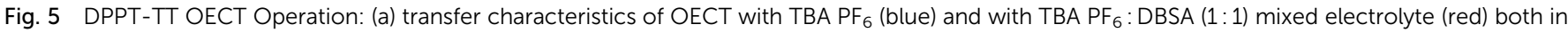
ACN; (b) transient gate voltage pulse of OECT with TBA PF 6 (blue) and with DBSA (red) added to the electrolyte mixture; (c) output characteristics with $\mathrm{TBA}^{\mathrm{P} F_{6}}$ electrolyte; (d) output characteristics with $\mathrm{TBA} \mathrm{PF}_{6}$ : DBSA (1:1) mixed electrolyte.

only to DPPT-TT but also PBTTT, where it appears to have an even stronger effect.

Fig. 6a shows the transfer characteristics of OECTs fabricated with PBTTT with the two different electrolytes. Without DBSA the ON current of the device is $\sim-35 \mu \mathrm{A}$ at a gate bias of $-0.8 \mathrm{~V}$ and the ON/OFF ratio is $\sim 10^{2}$ with an OFF current $\sim 10^{-1} \mu \mathrm{A}$. This is of relatively poor performance, but when DBSA is added to the gating electrolyte it has a huge effect on the ON current which increases to $-3.1 \mathrm{~mA}$ with no change in the ON/OFF ratio. The transconductance, $g_{m}$ increases from $0.05 \mathrm{mS}$ (no DBSA) to $3.9 \mathrm{mS}$ (with DBSA). Again, there is a significant decrease in the operation voltage with a drop in the $V_{\mathrm{ON}}$ value from $0.35 \mathrm{~V}$ to $0.12 \mathrm{~V}\left(\Delta V_{\mathrm{ON}}=0.23 \mathrm{~V}\right)$ (Fig. $\mathrm{S} 1$ for $I_{\mathrm{DS}}{ }^{1 / 2}$ plots, ESI $\dagger$ ). Adding DBSA also has a strong and positive effect on the transient gate bias pulse characteristics (Fig. 6b) as well as the output characteristics of PBTTT (Fig. $6 \mathrm{c}$ and $\mathrm{d}$ ): $I_{\mathrm{DS}}$ increases $\sim 25$ times from $\sim-140 \mu \mathrm{A}$ without DBSA to $\sim-3.3 \mathrm{~mA}$ with DBSA.
The highest performing PBTTT OECT device reported displays a transconductance $\approx 5 \mathrm{mS}, V_{\mathrm{ON}}=-0.35 \mathrm{~V}$, and an $\mathrm{ON}$ current of $\sim 0.15-0.20 \mathrm{~mA} .^{25}$ Although the device performance we report here without DBSA is relatively poor compared to the state-of-the-art, we manage to increase the transconductance of PBTTT to $3.9 \mathrm{mS}$, and achieve the highest drain current $(0.31 \mathrm{~mA}$ at $V=-0.8 \mathrm{~V})$ and lowest $V_{\mathrm{ON}}$ value $(0.12 \mathrm{~V})$ of any PBTTT device previously reported. This demonstrates the significance of DBSA in enhancing OECT device performance.

In summary, we demonstrate the benefit of the gate electrolyte mixture (TBA $\mathrm{PF}_{6}$ :DBSA) which improves device performance of OECTs by increasing the drain current and reducing the operation voltage. This is applicable to various conjugated polymers including P3HT, PBTTT, and DPPT-TT. Importantly, we unveil the operational mechanism of these conjugated polymer based OECT devices with mixed ion gating electrolyte showing a strong gate-modulated p-type doping/dedoping of the polymer. 

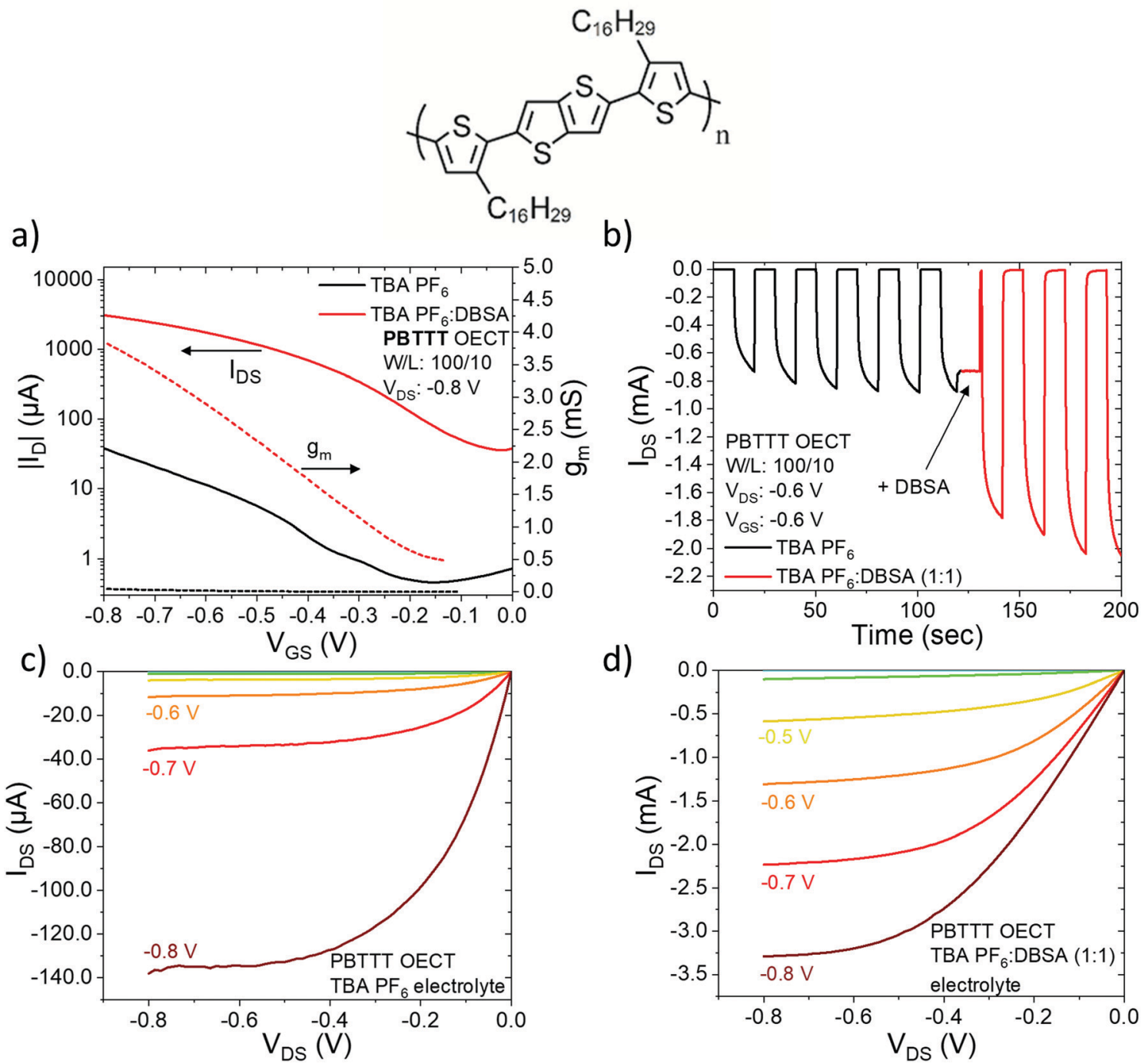

d)

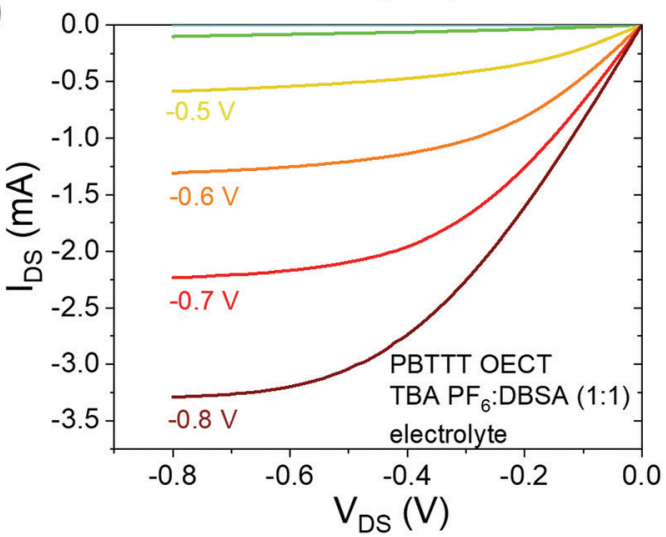

Fig. 6 PBTTT OECT Operation: (a) transfer characteristics of OECT with TBA PF 6 (black) and with TBA PF 6 : DBSA (1:1) mixed electrolyte (red) both in $A C N$; (b) transient gate voltage pulse of OECT with $\mathrm{TBA} \mathrm{PF}_{6}$ (black) and with DBSA (red) added to the electrolyte mixture; (c) output characteristics with $\mathrm{TBA}_{\mathrm{PF}} 6$ electrolyte; (d) output characteristics with $\mathrm{TBA}^{\mathrm{PF}} 6$ :DBSA electrolyte.

\section{Conclusions}

We present a small molecule additive, DBSA, that can be added to the gating electrolyte of accumulation mode OECTs resulting in lower operation voltage and higher ON currents, thus decreasing power consumption. We directly probe polaron formation in $\mathrm{P} 3 \mathrm{HT}$ via electrochemical doping to elucidate that the mixed $\mathrm{TBA} \mathrm{PF}_{6}$ :DBSA electrolyte generates polarons by the same mechanism (reversible electrochemical doping) as common electrolyte salts (e.g., TBA $\mathrm{PF}_{6}$ ). Importantly, polarons are generated at a lower potential and to a higher density. We confirm that no secondary doping effects on the polymer conformation or morphology take place. We apply the small molecule additive to other conjugated polymers, PBTTT and DPPT-TT for accumulation mode OECT operation. We observe the same effect and achieve an increase in the transconductance of PBTTT from $0.05 \mathrm{mS}$ to $3.9 \mathrm{mS}$ upon addition of DBSA.
Importantly, we observe a decrease in $V_{\mathrm{ON}}$ values for all polymers (PBTTT: 0.23 V, P3HT: $0.12 \mathrm{~V}$, DPPT-TT: $0.15 \mathrm{~V}$ ). Decreasing the operating-voltage of accumulation-mode OECTs, enables the implementation of higher performance p-type polymers with deeper HOMO levels as channel materials for OECTs, extending the repertoire of applications including biosensing. Interestingly, we find the behaviour of DBSA heavily dependent on the presence of other electrolyte salts. When alone, DBSA acts as an irreversible chemical dopant. When mixed with other salts however, (e.g. TBA $\mathrm{PF}_{6}$ ) it enhances the reversible electrochemical doping properties of the electrolyte. Thus, this small molecule additive can be used to dope the electrolyte and enhance device performance.

\section{Conflicts of interest}

There are no conflicts to declare. 


\section{Acknowledgements}

The authors acknowledge the UK EPSRC for studentships under DTG and the Plastic Electronics Centre for Doctoral Training (EP/L016702/1) and the Imperial College High Performance Computing Service for DFT calculations. This project has received funding from the European Research Council (ERC) under the European Union's Horizon 2020 research and innovation programme (grant agreement No. 723951). The author (E. Tan) thanks the National Physical Laboratory (NPL) for a CASE studentship.

\section{Notes and references}

1 A. Giovannitti, K. J. Thorley, C. B. Nielsen, J. Li, M. J. Donahue, G. G. Malliaras, J. Rivnay and I. McCulloch, Adv. Funct. Mater., 2018, 28, 1706325.

2 G. Tarabella, N. Coppedè, S. Iannotta, F. Cicoira, P. Kumar and C. Santato, Handbook of Organic Materials for Optical and (Opto)Electronic Devices: Properties and Applications, 2013, pp. 597-617.

3 A. M. Pappa, S. Inal, K. Roy, Y. Zhang, C. Pitsalidis, A. Hama, J. Pas, G. G. Malliaras and R. M. Owens, ACS Appl. Mater. Interfaces, 2017, 9, 10427-10434.

4 X. Strakosas, M. Bongo and R. M. Owens, J. Appl. Polym. Sci., 2015, 132, 1-14.

5 J. Rivnay, R. M. Owens and G. G. Malliaras, Chem. Mater., 2014, 26, 679-685.

6 D. T. Simon, E. O. Gabrielsson, K. Tybrandt and M. Berggren, Chem. Rev., 2016, 116, 13009-13041.

7 J. C. Carlberg and O. Inganäs, J. Electrochem. Soc., 1997, 144, 5-8. 8 A. Gölzhäuser, Phys. Chem. Chem. Phys., 2010, 12, 4273-4274.

9 D. Moia, A. Giovannitti, A. A. Szumska, I. P. Maria, E. Rezasoltani, M. Sachs, M. Schnurr, P. R. F. Barnes, I. McCulloch and J. Nelson, Energy Environ. Sci., 2019, 12, 1349-1357.

10 S. Muench, A. Wild, C. Friebe, B. Häupler, T. Janoschka and U. S. Schubert, Chem. Rev., 2016, 116, 9438-9484.

11 A. H. D. Macinnes, M. Druy, P. Nigrey, D. Nairns and A. McDiarmid, J. Chem. Soc., Chem. Commun., 1981, 317-319.

12 T. B. Schon, B. T. McAllister, P. F. Li and D. S. Seferos, Chem. Soc. Rev., 2016, 45, 6345-6404.

13 P. Gkoupidenis, D. A. Koutsouras and G. G. Malliaras, Nat. Commun., 2017, 8, 1-8.

14 Y. Van De Burgt, A. Melianas, S. T. Keene, G. Malliaras and A. Salleo, Nat. Electron., 2018, 1, 386-397.

15 A. Giovannitti, D. T. Sbircea, S. Inal, C. B. Nielsen, E. Bandiello, D. A. Hanifi, M. Sessolo, G. G. Malliaras, I. McCulloch and J. Rivnay, Proc. Natl. Acad. Sci. U. S. A., 2016, 113, 12017-12022.

16 A. Giovannitti, I. P. Maria, D. Hanifi, M. J. Donahue, D. Bryant, K. J. Barth, B. E. Makdah, A. Savva, D. Moia, M. Zetek, P. R. F. Barnes, O. G. Reid, S. Inal, G. Rumbles, G. G. Malliaras, J. Nelson, J. Rivnay and I. McCulloch, Chem. Mater., 2018, 30, 2945-2953.

17 T.-H. Le, Y. Kim and H. Yoon, Polymers, 2017, 9, 150.
18 L. Kergoat, B. Piro, M. Berggren, G. Horowitz and M. C. Pham, Anal. Bioanal. Chem., 2012, 402, 1813-1826.

19 Z. Yu, Y. Xia, D. Du and J. Ouyang, ACS Appl. Mater. Interfaces, 2016, 8, 11629-11638.

20 M. Yamashita, C. Otani, H. Okuzaki and M. Shimizu, Inst. Electr. Electron. Eng., 2011, 1, 3-6.

21 I. N. Hulea, H. B. Brom, A. J. Houtepen, D. Vanmaekelbergh, J. J. Kelly and E. A. Meulenkamp, Phys. Rev. Lett., 2004, 93, 16-19.

22 J. D. Yuen, A. S. Dhoot, E. B. Namdas, N. E. Coates, M. Heeney, I. McCulloch, D. Moses and A. J. Heeger, J. Am. Chem. Soc., 2007, 129, 14367-14371.

23 C. Pitsalidis, A. M. Pappa, M. Porel, C. M. Artim, G. C. Faria, D. D. Duong, C. A. Alabi, S. Daniel, A. Salleo and R. M. Owens, Adv. Mater., 2018, 30, 1-8.

24 A. M. Pappa, D. Ohayon, A. Giovannitti, I. P. Maria, A. Savva, I. Uguz, J. Rivnay, I. Mcculloch, R. M. Owens and S. Inal, Sci. Adv., 2018, 4, 1-8.

25 D. T. Duong, Y. Tuchman, P. Chakthranont, P. Cavassin, R. Colucci, T. F. Jaramillo, A. Salleo and G. C. Faria, Adv. Electron. Mater., 2018, 4, 1-7.

26 L. H. Jimison, S. A. Tria, D. Khodagholy, M. Gurfinkel, E. Lanzarini, A. Hama, G. G. Malliaras and R. M. Owens, Adv. Mater., 2012, 24, 5919-5923.

27 S. A. Tria, M. Ramuz, M. Huerta, P. Leleux, J. Rivnay, L. H. Jimison, A. Hama, G. G. Malliaras and R. M. Owens, Adv. Healthcare Mater., 2014, 3, 1053-1060.

28 H. Tang, F. Yan, P. Lin, J. Xu and H. L. W. Chan, Adv. Funct. Mater., 2011, 21, 2264-2272.

29 Q. Thiburce and A. J. Campbell, Adv. Electron. Mater., 2017, 3, 1600421.

30 S. Zhang, P. Kumar, A. S. Nouas, L. Fontaine, H. Tang and F. Cicoira, APL Mater., 2015, 3, 1-8.

31 S. Holliday, J. E. Donaghey and I. McCulloch, Chem. Mater., 2014, 26, 647-663.

32 Z. Chen, M. J. Lee, R. Shahid Ashraf, Y. Gu, S. AlbertSeifried, M. Meedom Nielsen, B. Schroeder, T. D. Anthopoulos, M. Heeney, I. McCulloch and H. Sirringhaus, Adv. Mater., 2012, 24, 647-652.

33 J. Nightingale, J. Wade, D. Moia, J. Nelson and J. Kim, J. Phys. Chem. C, 2018, 122, 29129-29140.

34 I. D. Baikie, A. C. Grain, J. Sutherland and J. Law, Energy Procedia, 2014, 60, 48-56.

35 M. Skompska, Electrochim. Acta, 1998, 44, 357-362.

36 M. Skompska and A. Szkurlat, Electrochim. Acta, 2001, 46, 4007-4015.

37 A. M. Gaikwad, Y. Khan, A. E. Ostfeld and S. Pandya, Org. Electron., 2016, 30, 18-29.

38 G. Zhang, M. Mcbride, N. Persson, S. Lee, T. J. Dunn, M. F. Toney, Z. Yuan, Y. Kwon, P. Chu, B. Risteen and E. Reichmanis, Chem. Mater., 2017, 29, 7645-7652.

39 W. C. Tsoi, D. T. James, J. S. Kim, P. G. Nicholson, C. E. Murphy, D. D. C. Bradley, J. Nelson and J. S. Kim, J. Am. Chem. Soc., 2011, 133, 9834-9843. 